Original article

\title{
Neutral lumbar spine sitting posture in pain-free subjects
}

\author{
Kieran O'Sullivan ${ }^{\mathrm{a}, *}$, Patrick O'Dea ${ }^{\mathrm{a}}$, Wim Dankaerts ${ }^{\mathrm{b}, \mathrm{c}}$, Peter O'Sullivan ${ }^{\mathrm{d}}$, Amanda Clifford ${ }^{\mathrm{a}}$, \\ Leonard O'Sullivan ${ }^{\mathrm{e}}$ \\ ${ }^{a}$ Physiotherapy Department, University of Limerick, Limerick, Ireland \\ ${ }^{\mathrm{b}}$ Musculoskeletal Unit, Department of Rehabilitation Sciences, Faculty of Kinesiology and Rehabilitation Sciences, Catholic University Leuven, Belgium \\ ${ }^{\mathrm{c}}$ Department of Health Care, Rehabilitation and Health Care Research Center, University College Limburg, Hasselt, Belgium \\ d School of Physiotherapy, Curtin University of Technology, Perth WA6845, Australia \\ ${ }^{\mathrm{e}}$ Department of Manufacturing and Operations Engineering, Physiotherapy Department, University of Limerick, Limerick, Ireland
}

\section{A R T I C L E I N F O}

\section{Article history:}

Received 8 January 2010

Received in revised form 4 May 2010

Accepted 14 June 2010

Keywords:

Posture

Sitting

Lumbar spine

\begin{abstract}
A B S T R A C T
Sitting is a common aggravating factor in low back pain (LBP), and re-education of sitting posture is a common aspect of LBP management. However, there is debate regarding what is an optimal sitting posture. This pilot study had 2 aims; to investigate whether pain-free subjects can be reliably positioned in a neutral sitting posture (slight lumbar lordosis and relaxed thorax); and to compare perceptions of neutral sitting posture to habitual sitting posture (HSP). The lower lumbar spine HSP of seventeen painfree subjects was initially recorded. Subjects then assumed their own subjectively perceived ideal posture (SPIP). Finally, 2 testers independently positioned the subjects into a tester perceived neutral posture (TPNP). The inter-tester reliability of positioning in TPNP was very good (intraclass correlation coefficient $($ ICC $)=0.91$, mean difference $=3 \%$ of range of motion). A repeated measures ANOVA revealed that HSP was significantly more flexed than both SPIP and TPNP $(p<0.05)$. There was no significant difference between SPIP and TPNP $(p>0.05)$. HSP was more kyphotic than all other postures. This study suggests that pain-free subjects can be reliably positioned in a neutral lumbar sitting posture. Further investigation into the role of neutral sitting posture in LBP subjects is warranted.
\end{abstract}

(c) 2010 Elsevier Ltd. All rights reserved.

\section{Introduction}

Low back pain (LBP) is a very common and costly musculoskeletal disorder (Woolf and Pfleger, 2003), with many different contributing factors including provocative spinal posture (Pynt et al., 2001; Pope et al., 2002; Scannell and McGill, 2003; Lis et al., 2007). While prolonged sitting in isolation is not a significant risk factor for developing LBP (Hartvigsen et al., 2000; Lis et al., 2007), combined exposure to prolonged sitting, awkward postures and vibration may increase the risk (Kelsey and Hardy, 1975; Lis et al., 2007). In addition, prolonged sitting is a common aggravating factor for many subjects with LBP (Williams et al., 1991; O'Sullivan, 2005). Considering the amount of time spent sitting in modern society, avoiding provocative seated spinal postures seems intuitively important (Li and Haslegrave, 1999).

The sitting posture of some LBP subjects differs to that of matched controls (Dankaerts et al., 2006b; Womersley and May,

\footnotetext{
* Corresponding author. Physiotherapy Department, University of Limerick, Ireland. Tel.: +353 61 234119; fax: +353 61234251.

E-mail address: kieran.osullivan@ul.ie (K. O’Sullivan).
}

2006). Reversing these provocative postures may help reduce LBP, and this is commonly advocated in LBP management (Poitras et al., 2005). There is still considerable debate on what the optimal seated lumbar posture is (Pynt et al., 2001; O'Sullivan, 2005; Claus et al., 2009a). Sitting postures do not all have the same effect on spinal load and trunk muscle activation (Adams and Hutton, 1985; O'Sullivan et al., 2002; Scannell and McGill, 2003; O'Sullivan et al., 2006a; Snijders et al., 2008; Claus et al., 2009b). Spinal flexion can negatively affect spinal proprioception (Dolan and Green, 2006), and can be associated with LBP (Womersley and May, 2006). Addressing these flexed sitting postures can reduce LBP, with many authors recommending lordotic seated postures to reduce pain (Williams et al., 1991; Lengsfeld et al., 2000; Womersley and May, 2006; Bettany-Saltikov et al., 2008; Pynt et al., 2008). Conversely, some studies report increased lordosis in LBP subjects (Christie et al., 1995; Vergara and Page, 2002; Dankaerts et al., 2006b; Van Dillen et al., 2009). Consistent with this, some LBP subjects report reduced pain with lumbar flexion (O'Sullivan, 2005), possibly related to mechanical and nutritional advantages of flexed postures (Adams and Hutton, 1985), relaxation of 
trunk muscles (O’Sullivan et al., 2006b) and unloading of spinal structures sensitised to extension loading (O'Sullivan, 2005).

It has been proposed that an optimal sitting posture for LBP subjects who are sensitised to flexion or extension is a more neutral spine position involving slight lumbar lordosis and a relaxed thorax (O'Sullivan et al., 2006a). This neutral posture avoids potentially painful end-range positions (Scannell and McGill, 2003), as well as activating key trunk muscles (O'Sullivan et al., 2006a; Claus et al., 2009b; Reeve and Dilley, 2009). However, assuming such a posture may be difficult to adopt (Claus et al., 2009a), questioning its application in clinical practice. The ability to reliably position subjects into a neutral lumbar spine sitting posture has not previously been assessed.

There is evidence of altered proprioceptive awareness in NSCLBP subjects (Brumagne et al., 2000; O'Sullivan et al., 2003). Appropriate performance of postural correction exercises requires an accurate perception of the instructed neutral posture. Therefore, subjective impressions of neutral sitting are important. Although there is evidence that subjects with neck pain display an altered sense of optimal posture (Edmondston et al., 2007; Falla et al., 2007), the perception of neutral posture among NSCLBP subjects has not been studied.

Thus, as a precursor to future studies on NSCLBP subjects, it is important to investigate if subjectively perceived ideal posture (SPIP) and an independent tester perceived neutral posture (TPNP) differ compared to the subject's habitual sitting posture (HSP) in healthy asymptomatic subjects. Such a comparison has not previously been reported. Thus the aim of this study was to investigate the inter-tester reliability of positioning subjects into a neutral sitting posture, and to compare SPIP and TPNP to HSP.

\section{Methods}

\subsection{Study design}

A single session, repeated measures study. The testers were two 4th year physiotherapy students. Prior to testing, the testers received training on positioning subjects into a neutral lumbar spine sitting posture from a musculoskeletal physiotherapist with 10 years clinical experience. This neutral posture aimed to position the lower lumbar spine into slight anterior pelvic tilt and slight lumbar lordosis, while maintaining relaxation of the thoracic spine. Inexperienced testers were used to evaluate if the TPNP could be instructed reliably, even in the absence of significant clinical experience.

\subsection{Subjects}

Seventeen participants ( 13 females) were recruited from within the university campus. Participants' mean $( \pm$ SD) age was $21( \pm 1)$ years, height was $170( \pm 7) \mathrm{cm}$, mass was $65( \pm 8) \mathrm{kg}$ and body mass index was $24( \pm 2) \mathrm{kg} / \mathrm{m}^{2}$. Participants were excluded if they had previous LBP, were on any current pain medications, or had undertaken previous postural control training. All participants provided written informed consent. Ethical approval was obtained from the local university research ethics committee.

\subsection{Instrumentation}

Postural data were collected using the Spinal Position Monitoring Device (SPMD) (“BodyGuard”, Sels Instruments, Belgium) (Fig. 1). This small wireless device monitors spinal sagital posture without cumbersome cables, facilitating more normal movement in a variety of tasks, inside and outside the laboratory. The SPMD incorporates a strain gauge that provides information about the relative distance between anatomical landmarks, calculating spinal flexion/

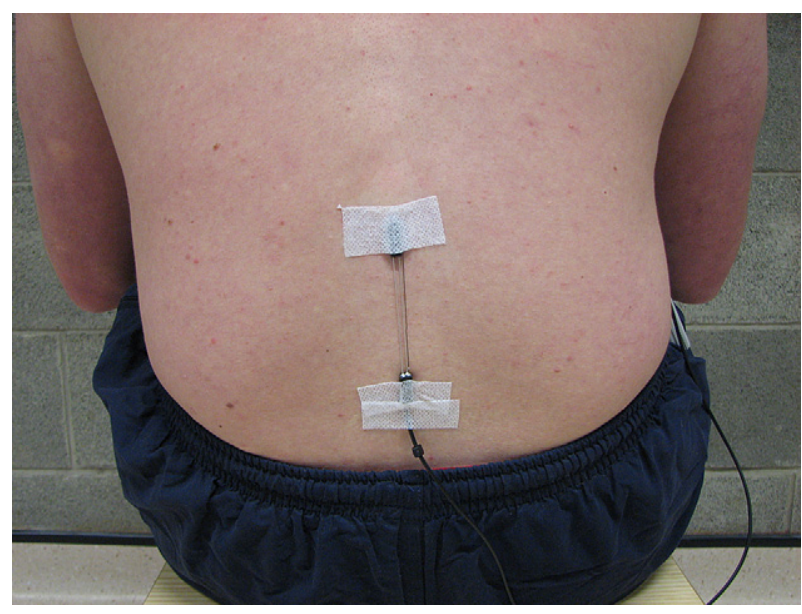

Fig. 1. SPMD

extension by the degree of strain gauge elongation. Subject posture is expressed as a percentage of strain gauge elongation, so that the degree of spinal flexion/extension is expressed relative to range of motion (ROM), rather than being expressed in degrees. Calculation of posture relative to ROM has been used in previous spinal posture research (Edmondston et al., 2007), and is similar to electromyography normalisation of muscle activity relative to sub-maximal voluntary contraction (Dankaerts et al., 2006a). Postural data was recorded in real-time at $20 \mathrm{~Hz}$. The SPMD has been shown to have very good reliability (intraclass correlation coefficient (ICC) $>0.8$ ) for the measurement of sitting posture (O'Sullivan et al., 2009).

\subsection{Procedure}

\subsubsection{Subject preparation}

During testing, participants sat on a wooden stool, wearing shorts, bare feet on the ground, knees shoulder width apart, and arms resting on their thighs. A $4 \mathrm{~cm}$ strain gauge was positioned directly over the spine at the spinal levels of L4 and S1, since the lower lumbar spine is the most common area for subjects to report NSCLBP (Dankaerts et al., 2006b). Recent research also suggests that the upper and lower lumbar spine regions demonstrate functional independence (Dankaerts et al., 2006b; Mitchell et al., 2008). The spinal levels of L4 and S1 were identified by manual palpation in a slightly flexed sitting posture. Once the SPMD was positioned, subjects performed maximal lumbar ROM to ensure the device was securely attached. To calibrate the SPMD, manual and verbal facilitation was used to guide subjects into a fully lordotic sitting posture which was set as $100 \%$ of their lumbar ROM (Fig. 2a), and then into a fully flexed sitting posture, which was set as $0 \%$ of their lumbar ROM (Fig. 2b). Thereafter, five trials of maximum extension and maximum flexion were performed to achieve a representative maximum lumbar ROM in sitting for each subject.

\subsubsection{HSP}

Immediately following calibration, subjects were instructed to 'sit as you usually do', while looking at a convenient fixed point straight ahead. During this time their HSP was recorded covertly for $1 \mathrm{~min}$, such that subjects were not informed that their HSP was being recorded, as per previous studies (Edmondston et al., 2007; Mitchell et al., 2008).

\subsubsection{SPIP}

Subjects were then asked to 'sit in a posture which you think is the ideal posture'. They were not given any other instructions, and did 


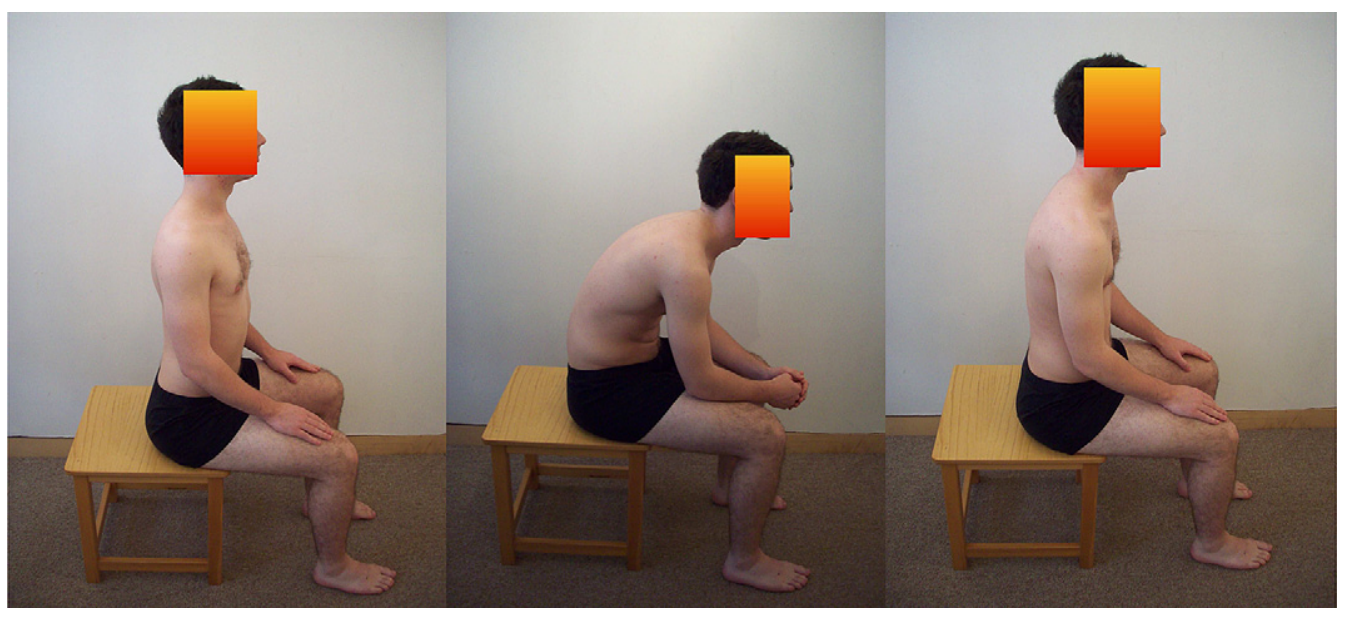

Fig. 2. Calibration to maximum extension and maximum flexion postures, and positioning in a neutral lumbar spine sitting posture, from left to right respectively.

not receive any feedback on their posture. This was held for $10 \mathrm{~s}$ and repeated five times, with a $10 \mathrm{~s}$ relaxation period in between each repetition. Both testers were blinded to the attempted SPIP by a screen placed between them and participants.

\subsubsection{TPNP}

Subjects were then facilitated into a neutral sitting posture by one tester (TPNP1) using manual and verbal facilitation (Fig. 2c). This posture was then held for $10 \mathrm{~s}$ and repeated five times with a $10 \mathrm{~s}$ period of relaxation in between repetitions, similar to the SPIP procedure. Following a $1 \mathrm{~min}$ rest period the other tester repeated the same procedure (TPNP2). The testers were blinded to each others facilitation of TPNP by a screen.

\subsection{Data analysis}

Subjects' mean posture recorded over the 1 min period was taken as their HSP. Similar to previous studies (Edmondston et al., 2007; Claus et al., 2009a), data at the start or end of the other sustained postures were excluded, to reduce the risk of data contamination due to beginning or ending movements. Therefore, data were extracted for the middle $3 \mathrm{~s}$ of each sustained posture, and averaged over the five measurements for each posture.

Data were analysed using SPSS 15.0. The data were normally distributed (Kolmogorov-Smirnov, $p>0.05$ ). To assess the reliability of TPNP, a two-way mixed ICC and Bland and Altman methods were used (Bland and Altman 1986). A repeated measures ANOVA (with Bonferonni post hoc comparisons) compared the four sitting postures; HSP, SPIP, TPNP1, TPNP2. The alpha level for statistical significance was set at $p<0.05$.

\section{Results}

\subsection{Inter-tester reliability of positioning in a neutral sitting posture}

The inter-tester reliability for positioning subjects into a neutral sitting posture (TPNP) was very good (ICC $=0.91,95 \% \mathrm{CI}$ : $0.74 \rightarrow 0.97)$. The mean difference between testers was only $3.3 \%$ $(95 \%$ CI $6.6 \rightarrow-0.6)$ of lower lumbar ROM.

\subsection{Perceptions of optimal sitting posture}

HSP was significantly more flexed than all three other postures; SPIP, TPNP1, and TPNP2 $(p=0.001, p=0.007$ and $p=0.002$ respectively) (Fig. 3). There were no significant differences between any of the other sitting postures (all $p>0.05$ ), although SPIP was slightly more lordotic than both TPNP measurements.

\section{Discussion}

\subsection{Inter-tester reliability of positioning subjects into a neutral sitting posture}

The reliability of positioning pain-free subjects in a neutral lumbar spine sitting posture based on the ICC values obtained was "almost perfect" (Landis and Koch, 1977). Furthermore, the mean difference values indicated that there was only a relatively small degree of variation, and no bias, between testers. The reliability of TPNP was greater than that reported previously when determining lumbar lordosis from photography (Fedorak et al., 2003) and numerous other physiotherapy procedures (Hicks et al., 2003; Johansson, 2006; Luomajoki et al., 2007).

\subsection{Differences in sitting postures}

Although the HSP was significantly more flexed than all other postures, it was still an approximately mid-range posture, supporting the hypothesis that pain-free subjects do not habitually sit in end-range postures (Dankaerts et al., 2006b). Subgroups of NSCLBP subjects adopt near end-range provocative postures (Dankaerts et al., 2006b). Assuming more neutral mid-range postures may help normalise spinal loading and trunk muscle activation in these patients (O'Sullivan, 2005; O'Sullivan et al., 2006a), although we did not evaluate muscle activation in these

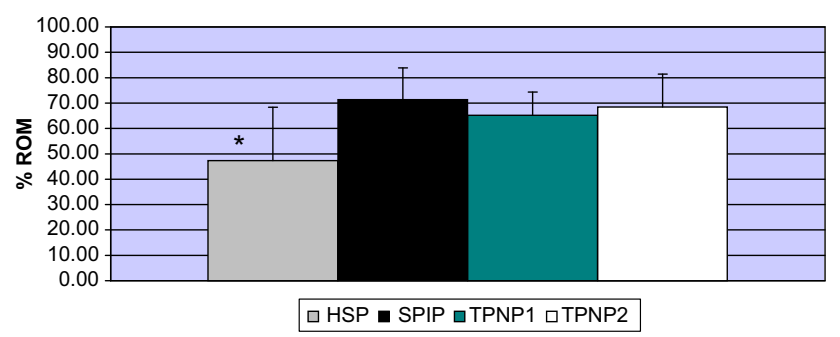

Fig. 3. Mean $( \pm \mathrm{SD})$ lumbar posture of all sitting postures; HSP, SPIP, and tester 1 and 2 perceived neutral postures (TPNP1 and TPNP2). All values expressed in \% of lumbar ROM, where $0 \%=$ full flexion and $100 \%=$ full extension. ${ }^{*}=$ HSP was significantly more flexed than all 3 other postures $(p<0.05)$ 
postures. Rehabilitation involving postural advice, as well as exercise, and relearning motor patterns, can modify lumbar posture such that it reduces passive tissue strain (Scannell and McGill, 2003) and pain (Dankaerts et al., 2007). However, a recent study suggested that even pain-free subjects may find assuming this neutral posture difficult without feedback (Claus et al., 2009a). We used both manual and verbal feedback to facilitate TPNP, to reflect clinical practice, as simply providing handouts may not be sufficient to facilitate neutral sitting postures. In addition, postural training would normally be accompanied by examination of the pain response of NSCLBP subjects, which may actually facilitate assuming TPNP more easily. It is encouraging that following only a brief period of training two relatively inexperienced testers could reliably position subjects in a neutral sitting posture.

There were no significant differences in lumbar posture between SPIP and TPNP. Other studies have shown that neutral spine sitting (TPNP in this study) activates key trunk muscles without significant activation of large, torque-producing muscles (O'Sullivan et al., 2006a; Claus et al., 2009b; Reeve and Dilley, 2009). This neutral posture also modifies activation of key thoracic and cervical muscle groups (Falla et al., 2007; Caneiro et al., 2010). This effect on muscle activation, although not measured in the current study, is important as spinal posture needs sufficient muscle activation to aid postural stability, without excess muscle activation imposing large compressive penalties and leading to fatigue (Gardner-Morse and Stokes, 1998; Granata and Marras, 2000; McGill et al., 2003; Kavcic et al., 2004; Claus et al., 2009b). SPIP was not significantly different to TPNP, although it was slightly more lordotic. During testing SPIP was often associated with thoracic extension, however these thoracic observations cannot be quantified. Since even minor changes in spinal sagital alignment can significantly alter trunk muscle activation (O'Sullivan et al., 2006a; Claus et al., 2009b; Reeve and Dilley, 2009), these variations in posture may be associated with significantly different levels of spinal load and are worthy of further study.

We acknowledge that the neutral sitting posture used in this study has not demonstrated superiority to other sitting postures in clinical trials. There is broad agreement that sitting involves more flexion than standing (Scannell and McGill, 2003; Claus et al., 2009a; Dunk et al., 2009; De Carvalho et al., 2010), but disagreement on how much flexion this should involve (Claus et al., 2009b). It is possible that advice on an optimal sitting posture will differ between subgroups with NSCLBP (O'Sullivan, 2005; Dankaerts et al., 2009).

There are few, if any, published studies on subjective impressions of optimal posture in NSCLBP. Edmondston et al. (2007) found no significant differences in cervical HSP in neck pain subjects compared to healthy controls, but interestingly SPIP differed significantly between the groups. Another study on neck pain subjects (Falla et al., 2007) demonstrated that subjective perceptions of optimal posture differed from physiotherapist perceptions, although that study quantified muscle activation and not spinal posture. While the current study involved only pain-free subjects, if differences between SPIP and TPNP are present in NSCLBP subjects, this may have implications for rehabilitation.

Finally, there is no suggestion that any sitting posture must always be maintained, as this could result in fatigue, discomfort and pain (Magnusson and Pope, 1998; Kavcic et al., 2004; Claus et al., 2009b). Instead, a neutral sitting posture may load the spine well during static low-load tasks, while allowing for normal movement. The ability to vary posture, so that neither rigid upright nor passive slumped postures are sustained, may help minimise the pain some NSCLBP subjects experience in sitting.

\subsection{Limitations}

The sample size was small, but similar to previous postural studies (O'Sullivan et al., 2002; Claus et al., 2009a, b). The subjects were primarily young and female, and habitual posture may vary across age and gender. The level of agreement on TPNP may differ in NSCLBP subjects, although pain relief may act as a guide and actually improve reliability. Reliability may vary according to the experience and training of those involved, although excellent reliability was obtained in this study with inexperienced physiotherapy students.

Postural data were not expressed in degrees, although calculating lumbar posture relative to ROM is useful in NSCLBP research (Dankaerts et al., 2006b). The reliability of identifying spinal levels was not assessed. Similar to all skin mounted systems, the SPMD does not directly calculate spinal posture. Despite this, any error in spinal posture would be consistent across all postures measured. Only lower lumbar posture was calculated. Measurement of upper lumbar and thoracic movement patterns should be studied in the future.

Only sagital, unsupported sitting postures were analysed, and investigations of other planes and types of sitting posture are needed. Analysis of HSP in a laboratory setting has inherent limitations, but its significance has previously been demonstrated (Szeto et al., 2002; Dankaerts et al., 2006b; Mitchell et al., 2008). Using adhesive tape could have increased postural awareness; however this was consistent across all subjects and does not explain the differences observed.

While postural factors may be significant for subgroups of NSCLBP subjects (Dankaerts et al., 2006b), it is acknowledged that NSCLBP should be considered within a biopsychosocial framework where numerous factors other than posture and movement patterns must be considered (McCarthy et al., 2004; Linton et al., 2007).

\section{Conclusion}

Pain-free subjects can be reliably positioned in a neutral lumbar spine sitting posture. In this current study HSP was significantly more flexed than both SPIP and TPNP. There was no significant difference between any of the other postures. Further research to investigate this neutral sitting posture in NSCLBP subjects is warranted.

\section{Acknowledgement}

The Health Research Board of Ireland funded the study. University of Limerick seed funding scheme facilitated the research collaboration. Colm Quinn, BSc (Physio) assisted with data collection.

\section{References}

Adams MA, Hutton WC. The effect of posture on the lumbar spine. Journal of Bone and Joint Surgery British Volume 1985;67(4):625-9.

Bettany-Saltikov J, Warren J, Jobson M. Ergonomically designed kneeling chairs are they worth it?: comparison of sagittal lumbar curvature in two different seating postures. Studies in Health Technology and Informatics 2008;140:103-6.

Bland JM, Altman DG. Statistical methods for assessing agreement between two methods of clinical measurement. Lancet 1986;1:307-10.

Brumagne SPT, Cordo PP, Lysens RMDP, Verschueren SP, Swinnen SP. The role of paraspinal muscle spindles in lumbosacral position sense in individuals with and without low back pain. Spine 2000;25(8):989-94.

Caneiro J, O'Sullivan P, Burnett A, Barach A, O'Neil D, Tveit O, et al. The influence of different sitting postures on head/neck posture and muscle activity. Manual Therapy 2010;1(15):54-60.

Christie H, Kumar S, Warren S. Postural aberrations in low back pain. Archives of Physical Medicine \& Rehabilitation 1995;76:218-24. 
Claus A, Hides J, Moseley G, Hodges P. Is “ideal” sitting real?: measurement of spinal curves in four sitting postures. Manual Therapy 2009a;14:404-8.

Claus AP, Hides JA, Moseley GL, Hodges PW. Different ways to balance the spine: subtle changes in sagittal spinal curves affect regional muscle activity. Spine 2009b;34(6):E208-14.

Dankaerts W, O'Sullivan P, Burnett A, Straker L. Altered patterns of superficial trunk muscle activation during sitting in nonspecific chronic low back pain patients: importance of subclassification. Spine 2006a;31(17):2017-23.

Dankaerts W, O'Sullivan P, Burnett A, Straker L. The use of a mechanism-based classification system to evaluate and direct management of a patient with nonspecific chronic low back pain and motor control impairment - a case report. Manual Therapy 2007;12(2):181-91.

Dankaerts W, O’Sullivan P, Burnett A, Straker L, Davey P, Gupta R. Discriminating healthy controls and two clinical subgroups of nonspecific chronic low back pain patients using trunk muscle activation and lumbosacral kinematics of postures and movements: a statistical classification model. Spine 2009·34(15):1610-8.

Dankaerts W, O'Sullivan PB, Burnett A, Straker LM. Differences in sitting postures are associated with non-specific chronic low back pain disorders when subclassified. Spine 2006b;31(6):698-704.

De Carvalho D, Soave D, Ross K, Callaghan J. Lumbar spine and pelvic posture between standing and sitting: a radiologic investigation including reliability and repeatability of the lumbar lordosis measure. Journal of Manipulative and Physiological Therapeutics 2010;33(1):48-55.

Dolan KJ, Green A. Lumbar spine reposition sense: the effect of a 'slouched' posture. Manual Therapy 2006;11(3):202-7.

Dunk N, Kedgley A, Jenkyn T, Callaghan J. Evidence of a pelvis-driven flexion pattern: are the joints of the lower lumbar spine fully flexed in seated postures? Clinical Biomechanics 2009;24(2):164-8.

Edmondston S, Chan H, Chi Wing Ngai G, Warren M, Williams J, Glennon S, et al Postural neck pain: \$n investigation of habitual sitting posture, perception of 'good' posture and cervicothoracic kinaesthesia. Manual Therapy 2007;12 (4):363-71.

Falla D, O'Leary S, Fagan A, Jull G. Recruitment of the deep cervical flexor muscles during a postural-correction exercise performed in sitting. Manual Therapy 2007; 12:139-43.

Fedorak CMD, Ashworth NMMF, Marshall JBM, Paull HDC. Reliability of the visual assessment of cervical and lumbar lordosis: how good are we? Spine 2003;28 (16):1857-9.

Gardner-Morse MG, Stokes IAF. The effects of abdominal muscle coactivation on lumbar spine stability. Spine 1998;23(1):86-91.

Granata KP, Marras WS. Cost-Benefit of muscle cocontraction in protecting against spinal instability. Spine 2000;25(11):1398-404.

Hartvigsen J, Leboeuf-Yde C, Lings S, Corder EH. Is sitting-while-at-work associated with low back pain? A systematic, critical literature review. Scandinavian Journal of Public Health 2000;28(3):230-9.

Hicks G, Fritz J, Delitto A, Mishock J. Interrater reliability of clinical examination measures for identification of lumbar segmental instability. Archives of Physical Medicine \& Rehabilitation 2003;84(12):1858-64.

Johansson F. Interexaminer reliability of lumbar segmental mobility tests. Manua Therapy 2006;11(4):331-6.

Kavcic N, Grenier S, McGill SM. Determining the stabilising role of individual torso muscles during rehabilitation exercises. Spine 2004;29(11):1254-65.

Kelsey J, Hardy R. Driving motor vehicles as risk factor for acute herniated lumbar intervertebral disc. American Journal of Epidemiology 1975;102:63-73.

Landis J, Koch G. The measurement of observer agreement for categorical data. Biometrics 1977;33:159-74.

Lengsfeld M, Franka A, van Deursen D, Griss P. Lumbar spine curvature during office chair sitting. Medical Engineering and Physics 2000;22(9):665-9.

Li G, Haslegrave C. Seated work postures for manual, visual and combined tasks. Ergonomics 1999;42(8):1060-86.

Linton S, Boersma K, Jansson M, Overmeer T, Lindblom K, Vlaeyen J. A randomized controlled trial of exposure in vivo for patients with spinal pain reporting fear of work-related activities. European Journal of Pain 2007;12(6):722-30.

Lis A, Black K, Korn H, Nordin M. Association between sitting and occupational LBP. European Spine Journal 2007;16:283-98.
Luomajoki H, Kool J, de Bruin ED, Airaksinen O. Reliability of movement control tests in the lumbar spine. BMC Musculoskeletal Disorders 2007;8:90.

Magnusson ML, Pope MH. A review of the biomechanics and epidemiology of working postures (it isn't always vibration which is to blame). Journal of Sound and Vibration 1998;215(4):965-76.

McCarthy C, Arnall F, Strimpakos N, Freemont A, Oldham J. The biopsychosocial classification of non-specific low back pain: a systematic review. Physical Therapy Reviews 2004;9(1):17-20.

McGill SM, Grenier S, Kavcic N, Cholewicki J. Coordination of muscle activity to assure stability of the lumbar spine. Journal of Electromyography and Kinesiology 2003;13:353-9.

Mitchell T, O'Sullivan P, Burnett AF, Straker L, Smith A. Regional differences in lumbar spinal posture and the influence of low back pain. BMC Musculoskeletal Disorders 2008;9:152.

O'Sullivan K, Galleotti L, Dankaerts W, O'Sullivan P, O'Sullivan L. The reliability of a wireless monitor for measurement of lumbar spine posture. Edinburgh, UK; 2009.

O'Sullivan P. Diagnosis and classification of chronic low back pain disorders: maladaptive movement and motor control impairments as underlying mechanism. Manual Therapy 2005;10(4):242-55.

O'Sullivan P, Burnett A, Floyd A, Gadsdon K, Logiudice J, Miller D, et al. Lumbar repositioning deficit in a specific low back pain population. Spine 2003;28:1074-9.

O'Sullivan PB, Dankaerts W, Burnett A, Farrell G, Jefford E, Naylor C, et al. Effect of different upright sitting postures on spinal-pelvic curvature and trunk muscle activation in a pain-free population. Spine 2006a;31(19):E707-12.

O'Sullivan PB, Dankaerts W, Burnett AF, Chen D, Booth R, Carlsen C, et al. Evaluation of the flexion relaxation phenomenon of the trunk muscles in sitting. Spine 2006b;31(17):2009-16.

O'Sullivan PB, Grahamslaw KM, Kendell M, Lapenskie SC, Moller NE, Richards KV. The effect of different standing and sitting postures on trunk muscle activity in a pain-free population. Spine 2002;27(11):1238-44

Poitras S, Blais R, Swaine B, Rossignol M. Management of work-related low back pain: a population-based survey of physical therapists. Physical Therapy 2005;85(11):1168-81.

Pope MH, Goh KL, Magnusson ML. Spine ergonomics. Annual Review of Biomedical Engineering 2002;4:49-68.

Pynt J, Higgs J, Mackey M. Seeking the optimal posture of the seated lumbar spine. Physiotherapy Theory \& Practice 2001;17(1):5-21.

Pynt J, Mackey MG, Higgs J. Kyphosed seated postures: extending concepts of postural health beyond the office. Journal of Occupational Rehabilitation 2008;18(1):35-45.

Reeve A, Dilley A. Effects of posture on the thickness of transversus abdominis in pain-free subjects. Manual Therapy 2009;14(6):679-84.

Scannell JP, McGill SM. Lumbar posture - should it, and can it, be modified? A study of passive tissue stiffness and lumbar position during activities of daily living. Physical Therapy 2003;83(10):907-17.

Snijders C, Hermans P, Niesing R, Kleinrensink G, Pool-Goudzwaard A. Effects of slouching and muscle contraction on the strain of the iliolumbar ligament. Manual Therapy 2008;13(4):325-33.

Szeto G, Straker L, Raine S. A field comparison of neck and shoulder postures in symptomatic and asymptomatic office workers. Applied Ergonomics 2002;33:75-84

Van Dillen L, Maluf K, Sahrmann S. Further examination of modifying patientpreferred movement and alignment strategies in patients with low back pain during symptomatic tests. Manual Therapy 2009;14(1):52-60.

Vergara M, Page A. Relationship between comfort and back posture and mobility in sitting-posture. Applied Ergonomics 2002;33:1-8.

Williams MM, Hawley JA, McKenzie RA, Van Wijmen PM. A comparison of the effects of two sitting postures on back and referred pain. Spine 1991; $16: 1185-91$.

Womersley L, May S. Sitting posture of subjects with postural backache. Journal of Manipulative and Physiological Therapeutics 2006;29(3):213-8.

Woolf A, Pfleger B. Burden of major musculoskeletal conditions. Bulletin of the World Health Organization; 2003. 